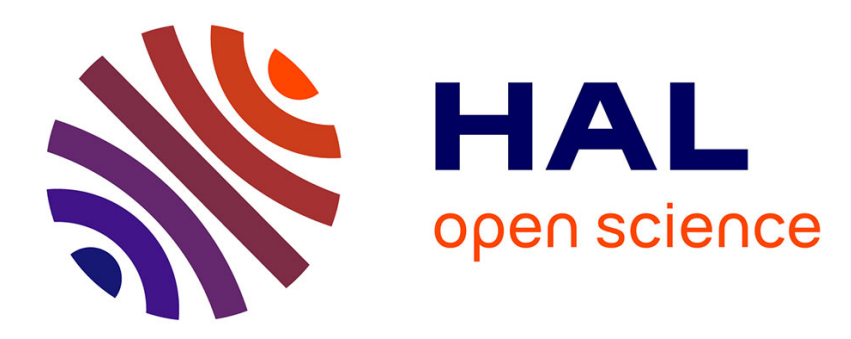

\title{
Parrainage et solidarités en basse Auvergne au XVI e siècle
}

\author{
Pierre-Yves Quémener
}

\section{To cite this version:}

Pierre-Yves Quémener. Parrainage et solidarités en basse Auvergne au XVI e siècle. Histoire, économie et société, 2018, Parrainage et compérage à l'époque moderne, 4. hal-03207480

\section{HAL Id: hal-03207480 \\ https://hal.science/hal-03207480}

Submitted on 25 Apr 2021

HAL is a multi-disciplinary open access archive for the deposit and dissemination of scientific research documents, whether they are published or not. The documents may come from teaching and research institutions in France or abroad, or from public or private research centers.
L'archive ouverte pluridisciplinaire HAL, est destinée au dépôt et à la diffusion de documents scientifiques de niveau recherche, publiés ou non, émanant des établissements d'enseignement et de recherche français ou étrangers, des laboratoires publics ou privés. 


\title{
Parrainage et solidarités en basse Auvergne au XVI ${ }^{\mathrm{e}}$ siècle
}

\author{
Pierre-Yves Quémener
}

\section{Résumé}

Les variables du parrainage (nombre de parrains et marraines, parrainage intrafamilial ou extrafamilial, transmission du nom aux filleuls) renseignent sur le type et le niveau des attentes des parents vis-à-vis de leurs compères en matière de solidarité. En Auvergne, l'analyse des registres de baptêmes $\mathrm{du} \mathrm{XVI} \mathrm{I}^{\mathrm{e}}$ siècle révèle une bipolarité entre le nord et le sud. Au sud, les besoins d'entraide sont assurés essentiellement par les proches parents, dans le cadre d'une solidarité familiale. Il semblerait que ce système social fut également en usage en Auvergne septentrionale à l'origine mais qu'il ait subi à la fin du Moyen Âge l'influence du système en usage dans les pays de langue d'oïl, davantage fondés sur les solidarités de voisinage.

\section{Abstract}

The variables of sponsorship (number of sponsors, endogamic or exogamic sponsors, transmission of the name to the godchildren) provide information on the type and level of expectations of the parents towards their friends in terms of solidarity. In Auvergne, the analysis of baptismal registers of the sixteenth century reveals bipolarity between the north and the south. In the South, the needs of mutual assistance are provided mainly by the close kinship, in the context of family solidarity. It would seem that this social system was also used in northern Auvergne at the beginning, but that it had undergone at the end of the Middle Ages the influence of the system used in the countries of language of oil, more based on solidarities of neighborhood.

À mesure que se développe l'étude du parrainage en Europe, on constate que pour la période qui s'étend du bas Moyen Âge au début de l'époque moderne, les pratiques pouvaient être très diverses selon les régions, qu'il s'agisse du modèle de parrainage (nombre et sexe des parents spirituels), de l'existence ou non de liens familiaux entre parents et compères, ou du degré de transmission du nom des parrains et marraines aux filleuls et filleules ${ }^{1}$. En l'état actuel de la recherche, il est encore trop tôt pour déterminer

1. Guido Alfani et Vincent Gourdon, «Spiritual kinship and godparenthood : an introduction », dans Spiritual Kinship in Europe, 1500-1900, dir. Guido Alfani et Vincent Gourdon, Basingstoke, Palgrave Macmillan, 2012, p. 1-43 ; Vincent Gourdon, « Le renouveau de l'histoire du parrainage aux époques moderne et contemporaine en 
précisément les causes de ces divergences. Elles peuvent tenir à la fois du milieu social et des pratiques culturelles, liées à une certaine conception de la famille et des solidarités. L'objet de cette étude est d'apporter une contribution à cette recherche.

Dans le cadre de la préparation d'une thèse de doctorat sur l'histoire du nom de baptême et des pratiques nominatives aux $X^{\mathrm{e}}$ et $\mathrm{XVI}^{\mathrm{e}}$ siècles ${ }^{2}$, j'ai constaté que les frontières linguistiques constituaient fréquemment en cette matière des points de rupture, révélateurs de la diversité des pratiques culturelles. En Bretagne par exemple, les filles étaient généralement nommées par les marraines en pays bretonnant (elles recevaient le nom de leur marraine) tandis qu'elles étaient plutôt nommées par leurs parrains en pays gallo (elles recevaient une forme féminisée du nom de leur parrain). Le même constat ressort de l'étude des registres paroissiaux francs-comtois et savoyards du XVI ${ }^{\mathrm{e}}$ siècle : les filles étaient nommées par les marraines dans la zone du parler franc-comtois, mais principalement par les parrains en pays franco-provençal. Par ailleurs, le nombre des parrains et marraines était généralement de trois en Bretagne jusqu'à la fin du XVI ${ }^{\mathrm{e}}$ siècle, mais cette pratique fut progressivement abandonnée après la publication des décrets du concile de Trente qui avait ordonné en 1563, dans l'une de ses dernières sessions, qu' " une personne seulement, homme ou femme, ou tout au plus un seul homme et une femme soient parrain ou marraine lors d'un baptême ${ }^{3}$ ». Dans les diocèses du pays bretonnant, ces consignes furent adoptées à partir des années 1570, mais en revanche leur application fut retardée jusqu'au début du XVII ${ }^{\mathrm{e}}$ siècle dans certains diocèses du pays gallo. Il y a tout lieu de croire que la résistance au nouveau système dans ces diocèses se justifiait par la permanence d'un besoin spécifique devenu obsolète ou pris en charge par d'autres opérateurs en pays bretonnant.

Le choix de la basse Auvergne comme observatoire des pratiques du parrainage au $\mathrm{XVI}^{\mathrm{e}}$ siècle est donc particulièrement intéressant car la région est traversée d'ouest en est par le «croissant linguistique » (voir la carte de la figure 1) défini par les linguistes comme un "groupement de parlers intermédiaires situés entre les dialectes d'oc et les dialectes d'oïl ${ }^{4} \gg$. Il s'agit de voir ici si ces pratiques étaient différentes de part et d'autre de cette zone de transition linguistique. Jusqu'en 2015, le territoire de la circonscription administrative de l'Auvergne était composé des quatre départements de l'Allier, du Puy-deDôme, du Cantal et de la Haute-Loire. Du point de vue historique, la province d'Auvergne

France », Obradoiro de Historia Moderna, 2015, 24, p. 23-48 ; Christof Rolker, « Pater spiritualis. La parenté spirituelle à la fin du Moyen Âge et au début de l'époque moderne », dans Formes et réformes de la paternité à la fin du Moyen Âge et au début de l'époque moderne, dir. Aude-Marie Certin, Frankfurt am Main, Peter Lang, 2016, p. 69-92 ; Will Coster, Baptism and Spiritual Kinship in Early Modern England, Aldershot, Ashgate, 2002; Michael Bennett, «Spiritual Kinship and the Baptismal Name in Traditional European Society », dans Studies on the Personal Name in Later Medieval England and Wales, dir. Dave Postles et Joel T. Rosenthal, Kalamazoo, Medieval Institute Publications, 2006 ; Christiane Klapisch-Zuber, « Parrains et filleuls, Étude comparative », dans La Maison et le Nom. Stratégies et rituels dans l'Italie de la Renaissance, Paris, Éditions de l'École des hautes études en sciences sociales, 1990, p. 109-122 ; Christian Maurel, « Prénomination et parenté baptismale du Moyen Âge à la Contre-Réforme. Modèle religieux et logiques familiales », Revue de l'histoire des religions, 1992, 209, p. 393-412 ; Anita Guerreau-Jalabert, « Spiritus et caritas. Le baptême dans la société médiévale », dans La Parenté spirituelle, dir. Françoise Héritier-Augé et Élisabeth Copet-Rougier, Paris, Éditions des archives contemporaines, 1995, p. 133-203; Bernhard Jussen, «Le parrainage à la fin du Moyen Âge : savoir public, attentes théologiques et usages sociaux », Annales, économies, sociétés, civilisations, 1992, 47 (2), p. 467-502.

2. Pierre-Yves Quémener, Le nom de baptême aux $X V^{e}$ et $X V I^{e}$ siècles en Bretagne. L'observatoire breton, thèse de doctorat sous la direction de Michel Nassiet, Université d'Angers.

3. Les conciles recuméniques. Les décrets de Trente à Vatican II, dir. Guiseppe Alberigo, Paris, Éditions du Cerf, 1994, t. II-2, p. 1539.

4. Guylaine Brun-Trigaud, «Les enquêtes dialectologiques sur les parlers du Croissant: corpus et témoins », Langue française, $n^{\circ}$ 93, 1992, p. 23. 
n'incluait toutefois pas au $\mathrm{XVI}^{\mathrm{e}}$ siècle le Bourbonnais (dont le territoire correspondait approximativement au département de l'Allier) ni le Velay (intégré à la province du Languedoc). Les circonscriptions religieuses étaient également différentes. Le diocèse de Bourges s'étendait sur toute la partie occidentale du département actuel de l'Allier tandis que la partie orientale relevait du diocèse de Clermont qui couvrait tout le Puyde-Dôme et la partie nord-ouest du Cantal. En outre, tout au nord, le diocèse de Nevers possédait quelques paroisses dans le Bourbonnais et le diocèse d'Autun en avait également une trentaine (archiprêtrés de Moulins-Yzeure et de Pierrefitte). Au sud, le diocèse de Saint-Flour occupait une grande partie du Cantal et la partie occidentale de la Haute-Loire dont la partie orientale dépendait du diocèse du Puy-en-Velay.

La carte de la figure 1 présente les pays traditionnels d'Auvergne dont les contours correspondent généralement aux dispositions du relief ou aux frontières naturelles, notamment à la rivière de l'Allier qui traverse toute la région depuis la Margeride jusqu'au Bourbonnais, irriguant la grande plaine centrale de la Limagne et séparant la Chaîne des Puys à l'ouest (Monts Dôme, Monts Dore) des massifs du Livradois et du Forez à l'est.

Les sources primaires utilisées sont les registres paroissiaux de baptêmes disponibles pour le $\mathrm{XVI}^{\mathrm{e}}$ siècle $^{5}$. Pour cette étude, qui porte sur les pratiques du parrainage autour du croissant linguistique, le corpus est constitué essentiellement de registres du Puy-de-Dôme et de l'Allier, complétés par quelques sondages et recherches dans différents registres des départements voisins (Cantal, Creuse, Loire, Aveyron).

Ces registres ont fait l'objet pour la plupart de dépouillements systématiques par les membres des associations généalogiques de l'Auvergne et du Bourbonnais, et l'on dispose aujourd'hui de relevés informatisés, présentés sous forme synthétique et contenant l'essentiel des données des actes de baptême (date des baptêmes, noms et sexes des baptisés, noms et surnoms des parents, noms et surnoms des parrains et marraines). Une cinquantaine de relevés a été réalisée, principalement pour le département actuel du Puyde-Dôme ${ }^{6}$. Je me suis fondé principalement sur trente-cinq de ces relevés, sélectionnés selon différents critères : situation géographique de la paroisse, taille du corpus, ancienneté des registres, exhaustivité des données ${ }^{7}$. De nombreux registres paroissiaux sont lacunaires entre 1570 et 1598 . Ils ont peut-être été détruits par les troupes huguenotes du capitaine Merle, particulièrement actives dans la région pendant les guerres de Religion, et ils ne seront souvent repris qu'après la pacification en $1598^{8}$.

Quelques registres contiennent un nombre très réduit d'actes qui à eux seuls ne peuvent être significatifs pour une étude statistique. Ils ont néanmoins été retenus lorsqu'ils permettaient de repérer la répartition géographique des différents modèles. Avant de

5. Les registres paroissiaux de l'Allier sont consultables en ligne sur le site http://recherche.archives.allier.fr, ceux du département du Puy-de-Dôme sur le site http://www.archivesdepartementales.puydedome.fr, ceux du Cantal sur le site http://archives.cantal.fr et ceux de la Haute-Loire sur le site http://www.archives43.fr.

6. Cette étude n'aurait pu être réalisée sans le concours du Centre généalogique et héraldique de l'Auvergne et du Vivarais (CGHAV) et la participation de plusieurs généalogistes auvergnats et bourbonnais. Je tiens ici à remercier plus particulièrement Alain Rossi et Henri Ponchon pour le CGHAV, Chantal Brunner, Christiane de Vriendt, Stéphane Batigne, Valérie Khun et Alain Antony.

7. Certains relevés ne mentionnent pas l'identité des parrains et marraines. Le corpus étudié représente un peu plus de 18000 actes dont 15000 environ pour le XVI ${ }^{\mathrm{e}}$ siècle.

8. Sur le capitaine Merle et les guerres de Religion en Auvergne, voir notamment André-Georges Manry, Histoire d'Auvergne, Clermont-Ferrand, Volcan, 1965, p. 159-160 ; Histoire de l'Auvergne, dir. André-Georges Manry, Toulouse, Privat, 1974, p. 273 ; Pierre Charbonnier, Histoire de l'Auvergne des origines à nos jours, Haute et Basse-Auvergne, Bourbonnais et Velay, Clermont-Ferrand, De Borée, 1999, p. 301 ; André Imberdis, Histoire des guerres religieuses en Auvergne pendant les XVI et XVII siècles, Paris, Delahays, 1855. 
Fig. 1 - Les pays d'Auvergne.

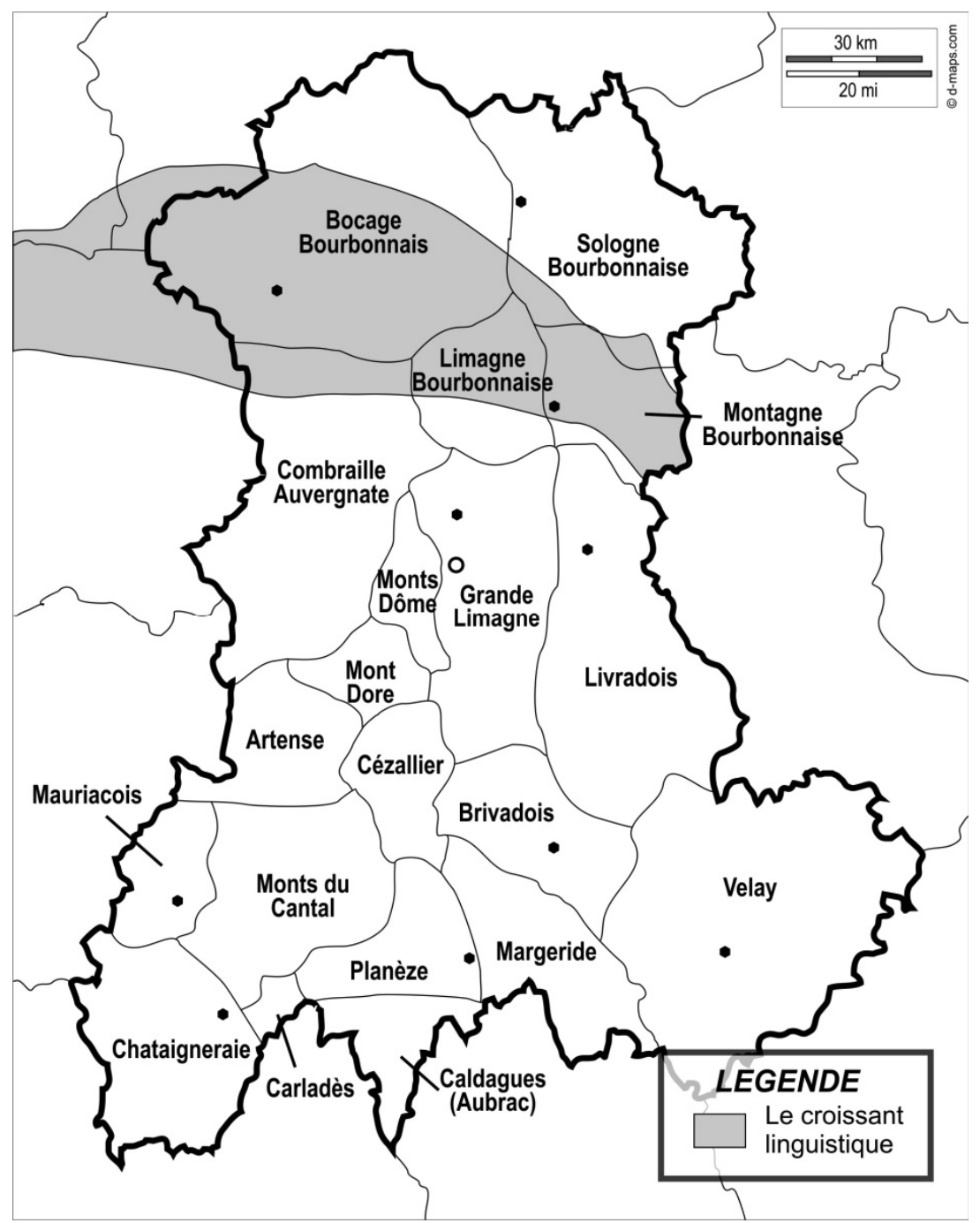

présenter les résultats de l'analyse statistique, il convient de rappeler brièvement quelles étaient les fonctions essentielles du parrainage au tournant du Moyen Âge et de l'époque moderne.

\section{Les fonctions du parrainage}

Pour l'Église catholique, le rôle des parrains et marraines est de garantir l'éducation religieuse de leurs filleuls et filleules. Il ne leur incombe pas de transmettre eux-mêmes cette instruction religieuse mais ils en sont responsables. Tertullien les qualifiait de « cautions » 
Tab. 1 - Les registres paroissiaux étudiés.

\begin{tabular}{|c|c|c|c|c|}
\hline Département & Paroisse & Période & $\begin{array}{c}\text { Nbre } \\
\text { d'actes }\end{array}$ & Lacunes \\
\hline \multirow{3}{*}{ Allier } & Melleray (Le Donjon) & $1539-1600$ & 897 & - \\
\hline & Thiel-sur-Acolin & $1563-1610$ & 576 & mars $72-$ avril 93 \\
\hline & Vaumas & $1546-1600$ & 698 & déc. 75 - févr. 88 \\
\hline \multirow{15}{*}{$\begin{array}{l}\text { Puy-de-Dôme } \\
\text { (ouest) }\end{array}$} & Saint-Georges-de-Mons & $1577-1605$ & 156 & juin 86 - juin 99 \\
\hline & Pontgibaud & $1550-1617$ & 1387 & - \\
\hline & Giat & $1570-1599$ & 120 & 1578-1599 \\
\hline & Herment & 1569-1599 & 69 & $1579-1598$ \\
\hline & Vernet Sainte-Marguerite & $1569-1603$ & 114 & juin $70-$ juin 98 \\
\hline & Saint-Nectaire & $1571-1630$ & 517 & mai 75 - juin 99 \\
\hline & Murol & $1577-1632$ & 49 & juin 77 - sept. 00 \\
\hline & Chambon-sur-Lac & $1572-1606$ & 167 & juin $78-$ juin 02 \\
\hline & Saurier & $1570-1607$ & 58 & nov. 73 - févr. 99 \\
\hline & Besse & 1537-1599 & 2481 & - \\
\hline & Bagnols & $1580-1629$ & 938 & mars $85-$ août 96 \\
\hline & Picherande & $1577-1613$ & 119 & août 78 - août 06 \\
\hline & Compains & 1569-1606 & 88 & avril $78-$ mai 00 \\
\hline & Ardes & $1563-1632$ & 517 & janv. $70-$ janv. 99 \\
\hline & Égliseneuve-d'Entraigues & $1569-1606$ & 250 & juin $75-$ janv. 00 \\
\hline Cantal & Condat & $1602-1630$ & 1307 & - \\
\hline \multirow{16}{*}{$\begin{array}{l}\text { Puy-de-Dôme } \\
\text { (est) }\end{array}$} & Charnat & $1567-1568$ & 13 & - \\
\hline & Noalhat & 1569-1607 & 43 & avril $73-$ nov. 99 \\
\hline & Chas & $1567-1630$ & 285 & août $70-$ janv. 00 \\
\hline & Chauriat & $1578-1625$ & 450 & avril $79-$ mai 89 \\
\hline & Billom & $1577-1625$ & 386 & sept. 86 - août 10 \\
\hline & Égliseneuve-près-Billom & $1580-1625$ & 283 & juil. 84 - janv. 04 \\
\hline & Glaine-Montaigut & $1569-1605$ & 115 & juin $78-$ janv. 99 \\
\hline & Augerolles & $1569-1625$ & 1448 & $1575-1598$ \\
\hline & Saint-Julien-de-Coppel & $1597-1628$ & 753 & - \\
\hline & Tours-sur-Meymont & $1577-1615$ & 1694 & - \\
\hline & Le Monestier & $1599-1630$ & 862 & - \\
\hline & Chambon-sur-Dolore & $1569-1607$ & 109 & mai 71 - sept. 99 \\
\hline & Saint-Anthème & $1570-1605$ & 169 & juil. $85-$ juin 01 \\
\hline & Saint-Clément de Valorgues & $1574-1630$ & 1087 & - \\
\hline & Saint-Romain & $1573-1604$ & 32 & juin $74-$ août 02 \\
\hline & Saillant & $1569-1602$ & 54 & mai 75 - juil. 01 \\
\hline
\end{tabular}

(sponsores), Augustin de « garants » (fidejussores $)^{9}$. Certains textes du XVI ${ }^{\mathrm{e}}$ siècle utilisent à l'occasion le terme de pleiges ${ }^{10}$. Les sources médiévales évoquent rarement cette fonction et il est difficile de dire dans quelle mesure elle était assumée par les parrains et marraines. On sait toutefois que les élites sociales ne s'en préoccupaient guère au début du XVIII siècle ${ }^{11}$.

Parallèlement à cette fonction religieuse, un parrain pouvait se sentir moralement responsable d'une assistance matérielle à l'égard de son filleul en cas de défaillance des parents. En 1561, Jean Calvin écrivait ainsi à son ami Nicolas des Gallars à propos du fils de son ancien médecin, Benoît Textor :

Le souvenir de son père doit me pousser à désirer qu'on aide ses enfants. Et j'ai cette raison particulière : de celui-là j'ai été le parrain ; il faut donc que je remplace son

9. François Babin, Conférences ecclésiastiques du diocèse d'Angers sur les Sacrements en général, sur le Baptême et la Confirmation, tenues en l'année 1716, Avignon, Giroud et Delorme, 1735, p. 293.

10. Voir par exemple Johann Boehme, Le recueil des pais selon leur situation avec les mours, loix et cérimonies d'iceux, Paris, Cavelier, 1558, p. 250 : «Iginius pape de Rome ordonna que si tost que l'enfant seroit né, on lui donnast des parrains qui seroient comme pleiges ou tesmoings de la foy pour ledit enfant. »

11. Ibid., p. 301 
père. [...] Je t'écris ceci pour que tu présentes ma recommandation à tous ceux dont il y aura lieu d'implorer la bonté en faveur de cet enfant. Que ma lettre en soit le témoin : quelque service qu'ils lui rendent, il me sera agréable à moi comme s’il était rendu à mon propre fils ${ }^{12}$.

Sans assimiler pour autant les parents spirituels à des «parents de remplacement», fonction plus généralement attribuée aux tuteurs désignés, en cas de décès des parents biologiques, il apparaît que les liens affectifs entre un parrain et son filleul amenaient naturellement le premier à se préoccuper du sort du second en cas de difficultés, tout au moins jusqu'à sa majorité ou son mariage : dans son testament daté de 1478, Antoine Pluver, changeur d'argent à Montpellier, lègue ainsi à son filleul la nourriture, les vêtements et le logement dans sa maison jusqu'à ce qu'il atteigne l'âge de 25 ans $^{13}$.

Socialement, le parrainage permettait surtout la constitution de réseaux de solidarité et d'amitié entre compères. Dans son registre d'inquisition rédigé de 1318 à 1325, l'évêque de Pamiers Jacques Fournier rapporte les propos de Guillaume Bélibaste pour qui le baptême et les compaternités «ne sont bons à rien, sinon à faire contracter des amitiés entre les hommes », ce qu'approuvait cependant Pierre Maury qui justifiait de cette façon les dépenses effectuées à l'occasion des fêtes du baptême car elles permettaient d'acquérir «l'amitié de beaucoup de personnes ${ }^{14}$ ». À vrai dire, la constitution de réseaux sociaux n'était pas une fonction primaire du parrainage, mais c'était implicitement le résultat attendu. La raison d'être de ces réseaux était vitale à la fin du Moyen Âge. Il ne s'agissait pas seulement de marquer l'appartenance à un groupe mais de satisfaire des besoins physiologiques élémentaires (la nourriture) ou des besoins de sécurité. Les réseaux de solidarités et d'assistance mutuelle permettaient de faciliter la vie quotidienne ou de se prémunir contre les aléas de l'existence. Les livres de famille des marchands florentins du $\mathrm{XV}^{\mathrm{e}}$ siècle apportent ainsi de nombreux exemples de services divers entre compères dans leurs activités ordinaires (médiations dans les transactions, prêts gracieux, etc.) ${ }^{15}$.

Christof Rolker estime que « la fonction sociale vraisemblablement la plus importante que les parrains exerçaient touchait à l'attribution du nom ${ }^{16} »$. Il s'agit en effet d'une caractéristique essentielle du parrainage, directement liée aux attentes en matière de solidarité. En acceptant d'être parrain, on s'engage, d'une part, à offrir des cadeaux de baptême à son filleul, d'autre part, à porter assistance à son compère en cas de besoin. En contrepartie, le père délègue au parrain l'honneur de la décision du nom de l'enfant et la faculté de lui transmettre son nom s'il le souhaite. Le nom de baptême n'est jamais donné au hasard, et cela est plus particulièrement vrai pour les noms de garçons. Il renvoie à un ou plusieurs référents que l'on veut honorer (le parrain, un membre de la parenté, un saint) et à qui l'on assigne implicitement la responsabilité de la protection de l'enfant. En ce sens, la transmission du nom du parrain au filleul est éminemment symbolique : le nom est alors

12. Émile Doumergue, Jean Calvin, Les hommes et les choses de son temps, Lausanne, Georges Bridel et $\mathrm{C}^{\text {ie }}, 1905$, t. III, p. 559.

13. Archives départementales de l'Hérault, 2 E 95/565, 5 juillet 1478, fol. 55. Je remercie Lucie Laumonier pour cette information et pour la communication de ses recherches sur les usages et fonctions de la parenté spirituelle en bas-Languedoc à la fin du Moyen Âge (communication personnelle du 5 mai 2017).

14. Jean Duvernoy, Le registre d'Inquisition de Jacques Fournier (évêque de Pamiers), 1318-1325, Paris, Mouton, 1978, 3 vol., t. I, p. 971, cité par B. Jussen, « Le parrainage à la fin du Moyen Âge », art. cit., p. 486.

15. Ch. Klapisch-Zuber, La Maison et le Nom, op. cit., p. 130, n. 38.

16. Ch. Rolker, « Pater spiritualis », art. cit., p. 72. 
un marqueur de la filiation spirituelle, il désigne publiquement devant toute la communauté celui qui s'est engagé moralement vis-à-vis des parents et de son filleul.

C'est à la lumière de ces engagements qu'il faut apprécier la diversité des pratiques du parrainage sachant que les marques d'honneur accordées aux parrains sont vraisemblablement proportionnelles aux bénéfices attendus.

\section{Les modèles de parenté baptismale}

Dans l'état actuel des recherches, il apparaît que trois modèles principaux de parrainage avaient cours en Europe occidentale à la fin du Moyen Âge ${ }^{17}$. Le « modèle du couple » (un parrain et une marraine par baptisé) dominait dans le Midi de la France, dans le sud de l'Allemagne et au nord de la Suisse ${ }^{18}$. Le «modèle ternaire » (deux parrains et une marraine pour un garçon, un parrain et deux marraines pour une fille) était le plus répandu en Bretagne, dans les pays français de langue d'oïl et dans le nord de l'Allemagne. En Allemagne centrale (environs de Francfort et Nuremberg), on se contentait toutefois d'un seul parrain ou d'une seule marraine (monoparrainage) ${ }^{19}$. On trouve également des communautés qui pratiquaient le multiparrainage illimité, par exemple en Italie du Nord, le nombre des parrains et marraines n'était pas toujours fixe et atteignait quelquefois une vingtaine de personnes ${ }^{20}$. En basse Auvergne, on observe une grande diversité de modèles de parrainage dans les registres de baptême du $X V I^{\mathrm{e}}$ siècle et de la première moitié du $\mathrm{XVII}^{\mathrm{e}}$ siècle avec des évolutions significatives dans certaines paroisses à la fin du XVI siècle.

En effet, outre le modèle du couple et le modèle ternaire présentés ci-dessus, on trouve dans certaines régions de la basse Auvergne un modèle ternaire très particulier où il n'y a toujours qu'un parrain et deux marraines, quel que soit le sexe de l'enfant. Pour le distinguer du modèle classique, je le désigne ici par l'expression « modèle ternaire féminin ». D'autre part, certaines paroisses avaient adopté un « modèle quaternaire pondéré » (deux parrains et deux marraines) tandis que d'autres utilisaient un « modèle quaternaire féminin », voire quinquénaire, à prédominance féminine (un parrain et trois ou quatre marraines quel que soit le sexe de l'enfant).

Les résultats sont synthétisés dans le tableau 2 et représentés schématiquement sur la carte de la figure 2. Sauf précision contraire, le modèle indiqué est le modèle dominant dans la paroisse (plus de $90 \%$ des occurrences). En général, lorsque le modèle dominant est celui du couple, le modèle minoritaire est le modèle ternaire, et inversement. Les premières dates indiquées correspondent à celles du début des registres. Lorsque le basculement entre le modèle ternaire et le modèle du couple s'est produit à une date précise et connue, elle est mentionnée sur le tableau. Les paroisses du diocèse de Clermont ont été classées à l'ouest ou à l'est en fonction de leur situation par rapport à l'Allier.

Il est possible de distinguer deux aires culturelles bien spécifiques.

17. Guido Alfani, Padri, padrini, patroni. La parentela spirituale nella storia, Venise, Marsilio, 2006, p. 65 ; G. Alfani et V. Gourdon, «Spiritual kinship and godparenthood : an introduction », art. cit., p. 7-8 ; V. Gourdon, « Le renouveau de l'histoire du parrainage », art. cit., p. 31-32 ; Ch. Rolker, « Pater spiritualis », art. cit., p. 73-75.

18. Pour la Suisse, voir Pierre Pegeot, « Un exemple de parenté baptismale à la fin du Moyen Âge, Porrentruy, 1482-1500), dans Les Entrées dans la vie. Initiations et apprentissages. XII congrès de la Société des historiens médiévistes de l'enseignement supérieur public, Nancy, Presses universitaires de Nancy, 1982, p. 53-70.

19. Ch. Rolker, « Pater spiritualis », art. cit., p. 73.

20. Christiane Klapisch-Zuber, «Compérage et clientélisme », dans La Maison et le Nom, op. cit., 1990, p. 126 ; Guido Alfani, « Les réseaux de marrainage en Italie du Nord du XV ${ }^{\mathrm{e}}$ au XVII ${ }^{\mathrm{e}}$ siècle : coutumes, évolution, parcours individuels », Histoire, économie et société, 2006, 4, p. 19-22. 
Tab. 2 - Modèles de parrainages au XVI ${ }^{\mathrm{e}}$ siècle et début du XVII ${ }^{\mathrm{e}}$ siècle

\begin{tabular}{|c|c|c|}
\hline Diocèse & Paroisse & Modèle de parrainage \\
\hline Clermont ouest & Besse & Couple depuis 1537. \\
\hline Clermont ouest & Ardes & Couple depuis 1563. \\
\hline Clermont est & Chas & Couple depuis 1567. \\
\hline Clermont ouest & $\begin{array}{l}\text { Egliseneuve- } \\
\text { d'Entraigues }\end{array}$ & Couple depuis 1569. \\
\hline Clermont ouest & Compains & Couple depuis 1569. \\
\hline Clermont est & Noalhat & Couple depuis 1569. \\
\hline Clermont est & Saillant & Couple depuis 1569. \\
\hline Clermont est & $\begin{array}{l}\text { Chambon sur } \\
\text { Dolore }\end{array}$ & $\begin{array}{l}\text { Couple de } 1569 \text { à } 1598 \text {. Ternaire féminin (60 \%) de } 1599 \\
\text { à } 1607 .\end{array}$ \\
\hline Clermont est & Glaine Montaigut & Couple $(70 \%)$ ou ternaire féminin (30\%) de 1569 à 1605. \\
\hline Clermont ouest & Saurier & Couple depuis 1570. \\
\hline Clermont est & Saint-Anthème & Couple depuis 1570. \\
\hline Clermont ouest & Saint-Nectaire & Couple depuis 1571. \\
\hline Clermont ouest & Chambon sur Lac & Couple depuis 1572. \\
\hline Clermont est & Saint-Romain & Couple depuis 1573. \\
\hline Clermont est & $\begin{array}{l}\text { Saint-Clément de } \\
\text { Valorgues }\end{array}$ & Couple depuis 1574. \\
\hline Clermont ouest & Murol & Couple depuis 1577. \\
\hline Clermont ouest & Picherande & Couple depuis 1577. \\
\hline Clermont est & Billom & Couple depuis 1577. \\
\hline Clermont est & $\begin{array}{l}\text { Tours sur } \\
\text { Meymont }\end{array}$ & Couple depuis 1577. \\
\hline Clermont est & Chauriat & Couple depuis 1578. \\
\hline Clermont ouest & Bagnols & Couple depuis 1580. \\
\hline Clermont est & $\begin{array}{l}\text { Égliseneuve près } \\
\text { Billom }\end{array}$ & $\begin{array}{l}\text { Couple (60 \%) ou ternaire féminin (40\%) de } 1580 \text { à } 1605 . \\
\text { Couple en } 1610 .\end{array}$ \\
\hline Clermont est & $\begin{array}{l}\text { Saint-Julien de } \\
\text { Coppel }\end{array}$ & Couple depuis 1597. \\
\hline Clermont ouest & Condat & Couple depuis 1574. \\
\hline Autun & $\begin{array}{l}\text { Melleray (Le } \\
\text { Donjon) } \\
\end{array}$ & Ternaire classique de 1539 à 1607 . Couple à partir de 1608. \\
\hline Clermont ouest & Vaumas & Ternaire classique de 1546 à 1601. \\
\hline Autun & Thiel sur Acolin & Ternaire classique de 1551 à 1623 . Couple à partir de 1624. \\
\hline Clermont est & Charnat & Ternaire $(46 \%)$ ou quaternaire $(54 \%)$ classique en $1567-1568$. \\
\hline Clermont est & Augerolles & $\begin{array}{l}\text { Ternaire féminin (69\%) en 1569-1570. Quaternaire (26\%) } \\
\text { de } 1606 \text { à } 1609\end{array}$ \\
\hline Clermont ouest & Pontgibaud & Ternaire classique de 1569 à 1612. Couple depuis avril 1612. \\
\hline Clermont ouest & Giat & $\begin{array}{l}\text { Ternaire classique de } 1570 \text { à } 1606 \text {. Quaternaire occasionnel } \\
(9 \%) \text {. }\end{array}$ \\
\hline Clermont ouest & Herment & $\begin{array}{l}\text { Ternaire classique de } 1571 \text { à } 1599 \text {. Quaternaire occ. Couple } \\
\text { depuis } 1600 .\end{array}$ \\
\hline Clermont est & Le Monestier & $\begin{array}{l}\text { Ternaire féminin de } 1599 \text { à } 1624 \text {. Couple à partir de } \\
\text { juillet } 1624 \text {. }\end{array}$ \\
\hline Clermont ouest & $\begin{array}{l}\text { Vernet Sainte- } \\
\text { Marguerite }\end{array}$ & Quaternaire pondéré de 1569 à 1603. \\
\hline Clermont ouest & $\begin{array}{l}\text { Saint-Georges de } \\
\text { Mons }\end{array}$ & Quaternaire pondéré de 1577 à 1605. \\
\hline
\end{tabular}

Dans toutes les paroisses méridionales du Puy-de-Dôme, le modèle du couple est quasiment exclusif depuis le début du XVI ${ }^{\mathrm{e}}$ siècle. Á Besse par exemple, les registres sont complets de 1537 à 1599 et on y relève seulement une dizaine de cas de modèles ternaires pour plus de 2500 baptêmes.

Dans le Cantal, les registres de Condat débutent en 1574 et le modèle ternaire n'y est pas usité. Il semblerait que la zone où le modèle du couple a toujours été la norme s'étendait au moins jusqu'au littoral méditerranéen puisque les registres de Millau (Rouergue), ouverts 
en 1534, ignorent pareillement le modèle ternaire ${ }^{21}$, tous comme ceux de Marseille, ouverts en $1510^{22}$.

Le modèle du couple est également la norme dans le sud-est du Puy-de-Dôme (SaintAnthème, Saint-Clément-de-Valorgues, Saint-Romain, Saillant), à Tours-sur-Meymont, à Sallèdes et généralement dans le pays de Billom (Billom, Chas, Chariat, Saint-Juliende-Coppel). Les registres de Chambon-sur-Dolore (1569-1607) présentent toutefois une évolution très particulière. De 1569 à 1571 , on n'y connaît que le modèle du couple comme dans les paroisses voisines du sud-Livradois. Les registres sont totalement lacunaires pendant les guerres de Religion de 1572 à 1598. À la reprise des registres, et jusqu'en 1607 , les paroissiens ont conservé pour partie le modèle du couple (36 baptêmes) mais une part importante d'entre eux a adopté le modèle ternaire féminin caractéristique du Livradois (54 baptêmes pour 26 garçons et 28 filles). L'adoption du modèle ternaire s'est produite également tardivement dans la ville voisine du Monestier en 1599. Il se développe parallèlement au modèle du couple, pour devenir quasiment exclusif à partir de 1603. Il restera la norme jusqu'en juillet 1624, avant d'être brutalement et définitivement abandonné.

La situation est très différente dans le Nord. Dans le Bourbonnais (Thiel, Vaumas, Le Donjon), la norme est clairement le modèle ternaire classique depuis le début du $\mathrm{XVI}^{\mathrm{e}}$ siècle. Le recours à plusieurs parrains ou marraines est également la norme jusqu'au début du $\mathrm{XVII}^{\mathrm{e}}$ siècle dans les paroisses de la Combraille auvergnate (Saint-Georges-de-Mons, Giat, Herment) et dans les Monts-Dôme (Pontgibaud, Le-Vernet-Sainte-Marguerite). L'usage systématique du modèle quaternaire pondéré à Saint-Georges-de-Mons et Le-Vernet-SainteMarguerite est particulièrement exceptionnel. Ce modèle est également utilisé fréquemment dans la Montagne Bourbonnaise (Charnat) et occasionnellement dans deux autres paroisses septentrionales du Puy-de-Dôme (Giat, Herment). Plus à l'ouest, dans le département actuel de la Creuse, le modèle ternaire classique était quasiment exclusif à Néoux dans les années 1573-1575. Ce modèle perdure au tournant du XVI ${ }^{\mathrm{e}}$ siècle et du XVII ${ }^{\mathrm{e}}$ siècle mais conjointement au modèle quaternaire pondéré, de la même manière que dans la Combraille auvergnate ${ }^{23}$.

Dans la partie nord du Livradois (Augerolles), la pratique dominante est par contre celle du modèle ternaire féminin. Ce modèle spécifique se retrouve également dans deux paroisses du secteur de Billom (Égliseneuve-près-Billom et Glaine-Montaigut), parallèlement au modèle du couple. Il semblerait que l'on se trouve ici dans une zone de transition entre le système septentrional qui privilégie le modèle ternaire et le système méridional qui privilégie le modèle du couple.

Les premiers registres de baptême d'Égliseneuve-près-Billom couvrent la période de 1580 à 1625 avec des lacunes totales entre 1585 et 1603 , puis entre 1606 et 1609 . Les mères ne sont jamais identifiées avant 1618. De 1580 à 1605, il y a 196 actes au total et l'on trouve sur toute cette période des baptêmes avec un parrain et une marraine (116 cas) et d'autres avec un parrain et deux marraines ( 79 cas pour 34 garçons et 45 filles). On relève également un baptême de garçon avec un parrain et trois marraines. Il n'y a pas de « marrainage intensif » par une deuxième marraine spécifique (sage-femme ou autre) ${ }^{24}$.

21. Les registres de l'Aveyron sont consultables en ligne sur le site http://archives.aveyron.fr/.

22. C. Maurel, « Prénomination et parenté baptismale », art. cit., p. 407.

23. Relevés de baptêmes de Néoux (1573-1792), transcriptions de Nathalie Bounaud, Pierre Bourut et Jean Peyne. Relevés mis en ligne sur le site http://www.gendep23.org/ [consulté le 20 mai 2017].

24. Les sages-femmes étaient toujours présentes aux cérémonies du baptême. Dans le Lyonnais, les registres de la paroisse de Cours du début du XVII ${ }^{\mathrm{e}}$ siècle mentionnent régulièrement les « bonnes mères » en même temps 
Fig. 2 - Les modèles de parrainage au XVI ${ }^{\mathrm{e}}$ siècle.

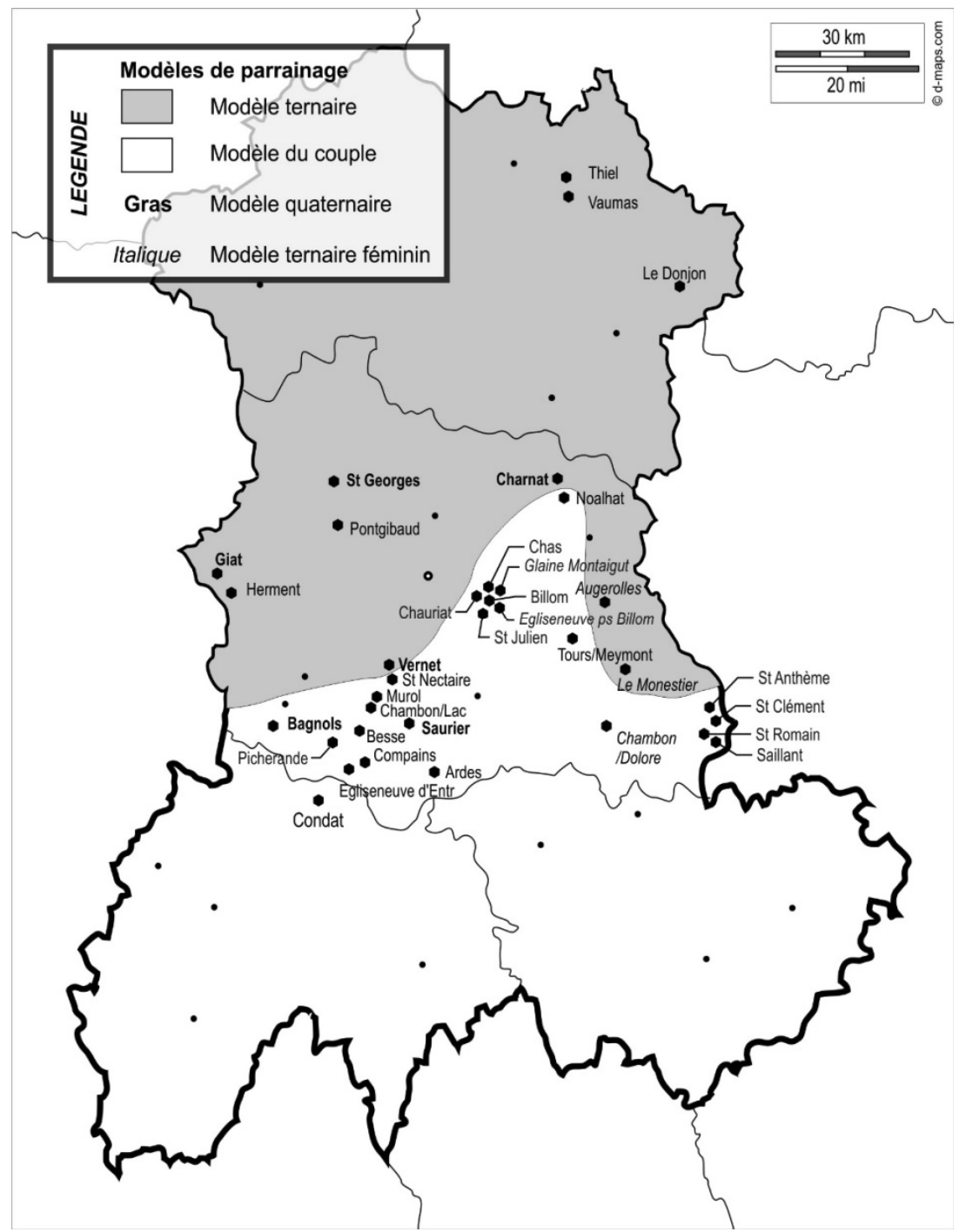

Il arrive cependant régulièrement que la seconde marraine porte le même patronyme que le père, ce qui laisserait penser qu'elles étaient sélectionnées plus fréquemment dans le milieu familial.

Les mêmes constats peuvent être dressés à Glaine-Montaigut (registres de 1569 à 1605), où l'on observe une coexistence entre le modèle du couple et le modèle ternaire féminin jusqu'en 1601. Pour la première période (1569-1601), on totalise 101 actes de baptême,

que les parrains et marraines. Le modèle dominant est celui du couple. Registres consultables en ligne sur le site des Archives départementales du Rhône : http://archives.rhone.fr (cote 66 GG 1) [consulté le 20 mai 2017]. 
dont 69 avec un parrain et une marraine et 32 avec un parrain et deux marraines (20 garçons et 12 filles).

Nous avons vraisemblablement dans toute la partie septentrionale du Puy-de-Dôme (à l'exception de la plaine de la Limagne auvergnate) une influence des pratiques habituelles dans le nord de la France où dominait le modèle ternaire avant la mise en application des recommandations tridentines ${ }^{25}$. Il y aurait eu acculturation progressive des populations de cette région, peut-être à la faveur d'une installation dans les massifs montagneux de migrants originaires de pays de langue d'oïl. En analysant les structures agraires de la Grande Limagne, le géographe Max Derruau soulignait justement le contraste frappant entre le nord et le sud. La Limagne bourbonnaise est caractérisée par un habitat dispersé, des grandes propriétés, des dialectes d'oïl. La Limagne auvergnate possède au contraire un habitat groupé, des exploitations de taille plus réduite et des parlers de langue d'oc ${ }^{26}$. L'auteur en concluait que le nord avait été occupé par une civilisation de défricheurs parlant une langue d'oïl, étroitement soumis à leurs seigneurs, tandis que la civilisation du sud, de langue d'oc, était moins nettement seigneuriale ${ }^{27}$. Les auteurs du recueil Structures agraires et paysages ruraux précisent pour leur part que :

Le sud a connu une occupation continue, avec subdivision en lanières de larges parcelles primitives par une série d'accensements seigneuriaux dans les derniers siècles du Moyen Âge ; au nord, au contraire, l'occupation semble avoir été interrompue, et la colonisation reprise tardivement, par des défrichements qu'effectuèrent de petits groupes familiaux, découpant des parcelles irrégulières ${ }^{28}$.

Il est possible par conséquent que le modèle du couple dominait primitivement dans la partie septentrionale du Puy-de-Dôme et que le modèle ternaire y ait été introduit au cours des campagnes de défrichements qui eurent lieu à la fin du Moyen Âge après les crises démographiques. Cette acculturation aurait produit par endroits des modèles originaux, de type quaternaire ou ternaire féminin comme on a pu le voir dans certaines paroisses. Le modèle ternaire spécifique observé dans le Livradois était par ailleurs également en usage à Chalmazel dans le Forez (département actuel de la Loire) à la fin du XVI ${ }^{\mathrm{e}}$ siècle $^{29}$, ainsi qu'à Rive-de-Gier dans le Lyonnais à la même époque ${ }^{30}$.

Dans toute la partie est du Puy-de-Dôme, nous avons observé des pratiques diverses et évolutives. Il semblerait que la pratique la plus ancienne dans ce secteur soit celle du modèle du couple mais qu'elle ait été délaissée dans certaines paroisses au profit du modèle ternaire très particulier du Livradois, octroyant aux marraines un rôle prépondérant dans les cérémonies du baptême. Plusieurs hypothèses explicatives peuvent être envisagées. On peut notamment s'interroger sur des liens éventuels avec les formes d'organisation sociale spécifiques (communautés taisibles et communautés de prêtres filleuls) qui se sont développées dans les régions montagneuses du Massif Central. Les communautés

25. V. Gourdon, «Le renouveau de l'histoire du parrainage », art. cit., p. 31. p. 405 .

26. Max Derruau, La grande Limagne auvergnate et bourbonnaise, Grenoble, Imprimerie Allier, 1949,

27. Ibid., p. 485.

28. E. Juillard, A. Meynier, X. de Planhol et G. Sautter, Structures agraires et paysages ruraux, Nancy, Annales de l'Est, 1957, p. 42.

29. Ambroise Jacquet, Recherches pour servir à l'histoire de Chalmazel, 1842, p. 31 du $1^{\mathrm{er}}$ manuscrit, transcription par Geneviève Adilon, mis en ligne sur le site http://forezhistoire.free.fr [consulté le 20 mai 2017].

30. Communication personnelle de Fabrice Montmartin du 18 mai 2017. 
taisibles, appelées également frérèches, parsonneries ou comparsonneries, sont apparues vers la fin du XIII ${ }^{\mathrm{e}}$ siècle, principalement dans des régions boisées ou bocagères défrichées tardivement. Il s'agissait de communautés familiales, parfois nombreuses, regroupées pour faciliter l'organisation du travail en commun, sous l'autorité d'un chef élu. La gestion domestique était administrée par une maîtresse qui n'était jamais l'épouse du maître mais qui jouait un rôle important dans la communauté ${ }^{31}$. Les communautés de prêtres filleuls se sont développées à la fin du Moyen Âge, principalement dans le centre de la France et en Bourgogne ${ }^{32}$. Elles étaient composées exclusivement de prêtres natifs de la paroisse $^{33}$ et avaient pour fonction principale de dire les messes des morts ${ }^{34}$, sur la base d'une formation religieuse le plus souvent rudimentaire ${ }^{35}$. Le prêtre filleul restait fortement attaché à sa famille et continuait de participer aux évènements familiaux. Il ne vivait pas nécessairement en communauté et pouvait choisir de rester au domicile familial ou dans un logement loué ${ }^{36}$. On sait que la mise en œuvre des recommandations tridentines a été particulièrement difficile dans le diocèse de Clermont, notamment en raison de la résistance au changement des prêtres filleuls qui souhaitaient conserver leurs prérogatives, fortement contestées au moment de la réforme catholique ${ }^{37}$. Communautés taisibles et communautés de prêtres filleuls marquent un attachement fondamental aux structures familiales. Il est possible que la vitalité du modèle du parrainage ternaire dans certaines paroisses (et sa résurgence dans d'autres) à la fin $\mathrm{du} \mathrm{XVI}^{\mathrm{e}}$ siècle soit liée à un souhait de consolidation des attaches familiales.

L'analyse des registres de baptême de la paroisse d'Augerolles au début du XVII ${ }^{\mathrm{e}}$ siècle amène toutefois à privilégier une autre hypothèse, de nature essentiellement économique. Dans cette paroisse le modèle ternaire féminin était prépondérant dès les premiers registres : en 1569-1570, il représente $69 \%$ des parrainages, contre $19 \%$ pour le modèle quaternaire féminin (un parrain et trois marraines) avec seulement $12 \%$ pour le modèle du couple ${ }^{38}$. En 1606, il ne représente toutefois plus que $15 \%$ des parrainages, à peine plus que le

31. La documentation sur les communautés taisibles est importante. On pourra se référer en particulier aux ouvrages suivants : Henriette Dussourd, Au même Pot et au même Feu... Étude sur les communautés familiales agricoles du centre de la France [1962], Paris, Maisonneuve et Larose, 1979; Bernard Derouet, « Système d'autorité et gestion des communautés familiales du Bourbonnais », Revue d'Auvergne, 1986, 100, p. 129-158 ; Jean Tricard, «Frérèches et comparsonneries à la fin du XV siècle : un exemple limousin », Revue d'Auvergne, 1986, 100, p. 119-127 ; Amable Chassaigne, Les communautés de famille en Auvergne, Paris, Rousseau, 1911.

32. Voir la carte publiée par Stéphane Gomis dans «Les communautés de prêtres sous l'Ancien Régime. Les acquis d'une redécouverte », Revue d'histoire de l'Église de France, 2000, 217, p. 470.

33. Ils devaient être nés et baptisés dans la paroisse et pour cette raison on les nommait filleuls car ils étaient à ce titre « fils de l'église à laquelle ils s'habituent ». Cf. Joseph-Nicolas Guyot, Répertoire universel et raisonné de jurisprudence, Paris, Visse, 1784, t. IV, p. 174 [article « Communaliste »].

34. Ibid., p. 476. Sur les communautés de prêtres, voir également Stéphane Gomis, Les « enfants prêtres » des paroisses d'Auvergne, XVI ${ }^{e}$ XVIII $I^{e}$ siècles, Clermont-Ferrand, Presses universitaires Blaise Pascal, 2006.

35. Louise Welter, «Les communautés de prêtres dans le diocèse de Clermont du XIII ${ }^{\mathrm{e}}$ au XVIII ${ }^{\mathrm{e}}$ siècle », Revue d'histoire de l'Église de France, 1949, 125, p. 16.

36. Ibid., p. 17 ; cf. J.-N. Guyot, Répertoire universel et raisonné de jurisprudence, op. cit., p. 174.

37. Les litiges portaient essentiellement sur les préséances et les revenus. Sur la difficile mise en œuvre de la réforme catholique, voir L. Welter, «Les communautés de prêtres », art. cit., p. 25-35 ; ead., La Réforme ecclésiastique du diocèse de Clermont au XVII ${ }^{e}$ siècle, Paris, Letouzey et Ané, 1956 ; Bernard Dompnier, «Clermont en 1665. Un diocèse à l'écart de la Réforme catholique ? », dans Fléchier et les Grands Jours d'Auvergne, dir. Emmanuèle Lesne-Jaffro, Tübingen, Gunter Narr, 2000, p. 33-53.

38. Relevés de baptêmes d'Augerolles aimablement transmis par Henri Ponchon (communication personnelle du 19 mai 2017) envers qui je suis grandement redevable pour cette étude sur Augerolles. Les registres paroissiaux de la collection du greffe sont consultables en ligne sur le site des Archives départementales du Puy-de-Dôme : http://www.archivesdepartementales.puydedome.fr mais cette collection est lacunaire de 1608 à 
modèle du couple $(10 \%)$, tandis que les modèles quaternaire $(34 \%)$ et quinquénaire (37\%) sont devenus prédominants (figure 3). Le modèle quinquénaire cesse en 1608, au profit du modèle ternaire. En 1610, c'est le modèle quaternaire qui cesse d'être utilisé mais en faveur cette fois du modèle du couple. Cet attrait inhabituel dans la paroisse pour le modèle du couple fera toutefois long feu puisqu'il est lui-même abandonné dès l'année suivante, portant du même coup la part du modèle ternaire à plus de $90 \%$. Il s'agit exclusivement de modèles à prépondérance féminine, c'est-à-dire avec un seul parrain et plusieurs marraines, indépendamment du sexe de l'enfant. En 1623, le modèle ternaire est lui-même abandonné définitivement, sans doute à la suite de consignes épiscopales car il s'interrompt brutalement dans plusieurs paroisses à la même époque : en 1624 au Monestier (diocèse de Clermont) ${ }^{39}$ et la même année à Thiel-sur-Acolin et à Luzy ${ }^{40}$ (diocèse d'Autun).

Fig. 3 - Modèles de parrainage à Augerolles de 1606 à 1625.

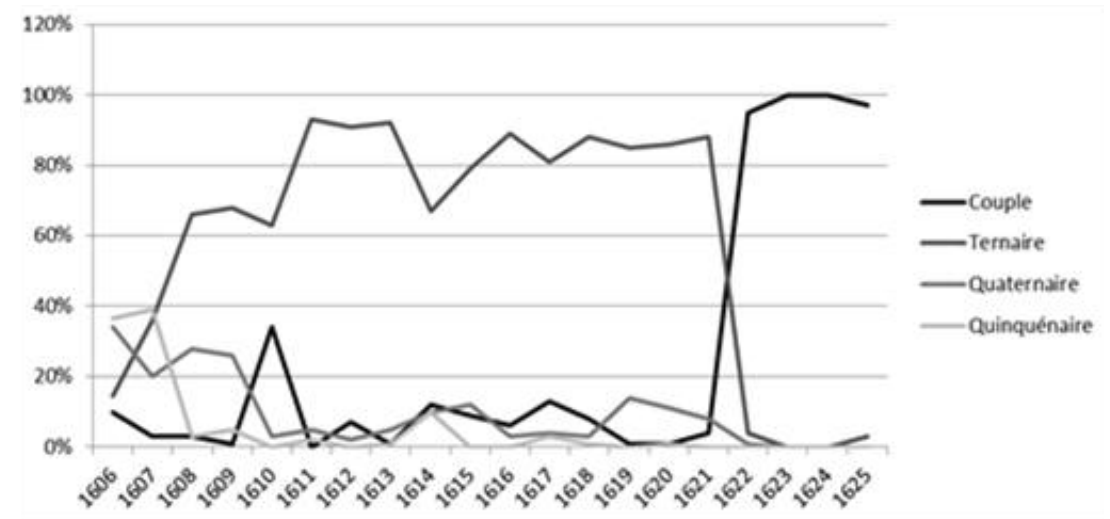

Qu'est-ce qui poussait les familles d'Augerolles à doter leurs enfants d'un nombre si important de marraines au début du XVII ${ }^{\mathrm{e}}$ siècle ? Une explication peut être avancée $a$ contrario à partir de l'examen des baptêmes pour lesquels il n'y avait qu'un seul parrain et une seule marraine. De 1607 à 1616, on comptabilise 27 baptêmes avec cette configuration, en excluant toutefois ceux de 1610, année où l'on a connu un accroissement exceptionnel du modèle du couple. Fait remarquable, dans 25 de ces actes, les parrains et marraines sont presque tous qualifiés par un titre honorifique : Honorable homme, Honorable femme, Honorable fille, Vénérable personne, Maître ou Messire, Noble, Damoiselle. Pour la plupart, ces hommes et ces femmes appartiennent à l'élite sociale de la communauté et sont issus du milieu des notaires et hommes de loi d'Augerolles ou des paroisses voisines ${ }^{41}$. Si la pratique du modèle du couple s'observe lorsque les parrains et marraines étaient financièrement

1667. Les statistiques présentées pour la période 1606-1625 ont été établies à partir des registres de la collection communale (relevés réalisés par Henri Ponchon).

39. Certaines paroisses du diocèse de Clermont avaient délaissé le modèle ternaire bien plus tôt : à Pontgibaud par exemple, il est abandonné une première fois au printemps 1608 mais rétabli dès le début de l'année suivante. Il ne sera définitivement abandonné qu'en avril 1612.

40. Lucien Gueneau, « Naissances, baptêmes, relevailles à Luzy », Mémoires de la Société académique du Nivernais, 1886, p. 41.

41. Communication personnelle d'Henri Ponchon du 19 mai 2017. 
aisés, on peut en déduire que le recours à deux, trois voire quatre marraines pouvait être la solution que l'on adoptait en cas d'insuffisance de ressources : l'accroissement du nombre de marraines permettait une répartition du coût du baptême entre un nombre plus important de contributrices ${ }^{42}$. Cette pratique était attestée dans les Alpes d'Ollon (Suisse romande) au début du $\mathrm{XX}^{\mathrm{e}}$ siècle où Louis Gauchat avait observé que " plusieurs marraines s'associent quelquefois pour donner leur présent en $\operatorname{commun}^{43} »$.

\section{La transmission du nom}

Si le nombre des parrains et marraines varie d'un territoire à l'autre, on observe en revanche partout le caractère normatif de la transmission du nom des parrains et marraines à leurs filleuls ou filleules. Je qualifie ce procédé de transmission homonymique.

Tab. 3 - Transmissions homonymiques au tournant du XVI et du XVII siècle

\begin{tabular}{|l|l|l|l|l|l|l|l|}
\hline \multicolumn{1}{|c|}{ Paroisse } & \multicolumn{1}{c|}{ Période } & parr. & $\begin{array}{c}\text { Garç. } \\
\text { marr. }\end{array}$ & Total & parr. & $\begin{array}{c}\text { Filles } \\
\text { marr. }\end{array}$ & Total \\
\hline Pontgibaud & $\begin{array}{l}1550- \\
1617\end{array}$ & $91 \%$ & $3 \%$ & $94 \%$ & $3 \%$ & $91 \%$ & $94 \%$ \\
\hline Giat & $\begin{array}{l}1570- \\
1599\end{array}$ & $79 \%$ & $5 \%$ & $84 \%$ & $3 \%$ & $72 \%$ & $75 \%$ \\
\hline Herment & $\begin{array}{l}1569- \\
1603\end{array}$ & $86 \%$ & - & $86 \%$ & - & $84 \%$ & $84 \%$ \\
\hline Besse & $\begin{array}{l}1537- \\
1599\end{array}$ & $95 \%$ & $1 \%$ & $96 \%$ & $3 \%$ & $94 \%$ & $97 \%$ \\
\hline Bagnols & $\begin{array}{l}1580- \\
1629\end{array}$ & $95 \%$ & $1 \%$ & $96 \%$ & $1 \%$ & $96 \%$ & $97 \%$ \\
\hline Ardes & $\begin{array}{l}1563- \\
1632\end{array}$ & $98 \%$ & - & $98 \%$ & - & $98 \%$ & $98 \%$ \\
\hline Condat & $\begin{array}{l}1602- \\
1630\end{array}$ & $99 \%$ & - & $99 \%$ & $1 \%$ & $96 \%$ & $97 \%$ \\
\hline Chauriat & $\begin{array}{l}1578- \\
1625\end{array}$ & $96 \%$ & $2 \%$ & $98 \%$ & - & $99 \%$ & $99 \%$ \\
\hline $\begin{array}{l}\text { Saint-Julien de } \\
\text { Coppel }\end{array}$ & $\begin{array}{l}1597- \\
1628\end{array}$ & $96 \%$ & $1 \%$ & $97 \%$ & $2 \%$ & $96 \%$ & $98 \%$ \\
\hline $\begin{array}{l}\text { Tours-sur- } \\
\text { Meymont }\end{array}$ & $\begin{array}{l}1577- \\
1615\end{array}$ & $92 \%$ & $3 \%$ & $95 \%$ & $34 \%$ & $52 \%$ & $86 \%$ \\
\hline Le Monestier & $\begin{array}{l}1599- \\
1630\end{array}$ & $95 \%$ & $2 \%$ & $97 \%$ & $12 \%$ & $84 \%$ & $96 \%$ \\
\hline Saint-Anthème & $\begin{array}{l}1570- \\
1605\end{array}$ & $86 \%$ & $1 \%$ & $87 \%$ & $5 \%$ & $87 \%$ & $92 \%$ \\
\hline $\begin{array}{l}\text { Saint-Clément } \\
\text { de Valorgues }\end{array}$ & $\begin{array}{l}1574- \\
1630\end{array}$ & $91 \%$ & $1 \%$ & $92 \%$ & $3 \%$ & $90 \%$ & $93 \%$ \\
\hline
\end{tabular}

Taux = proportion des garçons ou filles qui portent le nom de leurs parrains ou marraines.

42. Sur l'aspect financier du baptême, voir Guido Alfani et Vincent Gourdon, « Fêtes du baptême et publicité des réseaux sociaux en Europe occidentale. Grandes tendances de la fin du Moyen Âge au XX ${ }^{\mathrm{e}}$ siècle », Annales de démographie historique, 2009, 117, p. 153-189.

43. Louis Gauchat, La Trilogie de la vie, Articles-spécimens du Glossaire des patois de la Suisse romande, Lausanne, Imprimeries réunies, 1910, p. 10. 
Le tableau 3 totalise les taux de transmission des noms des parrains et marraines à leurs filleuls et filleules et précise si l'homonymie porte sur le nom des parrains ou des marraines. J'utilise l'expression attributions transversales lorsqu'un garçon reçoit une forme masculinisée du nom de sa marraine, ou lorsqu'une fille reçoit une forme féminisée du nom de son parrain. En cas de pluralité d'homonymies (ex. : le nom de la filleule est à la fois celui de la marraine et une forme féminisée du nom du parrain), je considère que l'homonymie s'est faite principalement avec la personne du même sexe.

Les taux de transmissions homonymiques sont relativement élevés par rapport à d'autres régions françaises où les taux évoluent généralement entre 70 et $90 \%$ à la même époque ${ }^{44}$. Il s'agit d'un indicateur particulièrement intéressant car il permet de « quantifier » l'honneur conféré aux parrains et marraines et, au-delà, de mesurer l'intensité des liens du compérage.

Dans les registres d'Ardes, les dérogations à la norme sont si exceptionnelles que le rédacteur ressent généralement le besoin de les expliciter. À plusieurs reprises, il précise par exemple que les parrains ou marraines désignés dans l'acte de baptême n'étaient pas ceux qui étaient pressentis initialement : en 1631, Anne Vialard est la marraine de Jacquette Vialard « au lieu de Jacquette Vialard, de Sauxillanges »; en 1631 également, Jean Nugier est parrain d'Antoine Nadaillat « au nom d'Antoine Nadaillat »; en 1632, Jeanne Carmantrand est la marraine d'Isabeau Pradelle « à la place d'Isabeau Anglade, sa fille », etc. On aura remarqué que dans ces actes, le nouveau-né n'a pas reçu le nom de son parrain ou de sa marraine mais celui de la personne qui avait vraisemblablement été sollicitée par les parents à l'origine et qui, faute de pouvoir assister à la cérémonie, a proposé un remplaçant sans que celui-ci puisse toutefois transmettre son nom. Il ne s'agit pas d'une délégation mais d'une véritable substitution. On peut penser néanmoins que le maintien de la référence nominative impliquait pour les personnes pressenties initialement un engagement vis-à-vis des parents ou de l'enfant sans que l'on puisse en préciser la teneur.

L'analyse des attributions transversales constitue un indicateur particulièrement intéressant car il permet de savoir qui était le nommeur principal - le parrain ou la marraine - lorsqu'il y avait transmission homonymique. Pour la nomination des garçons, il n'y a généralement pas de surprise : ce sont ici comme ailleurs les parrains qui transmettent leur nom à une très large majorité. L'analyse de la nomination des filles est par contre souvent très instructive car jusqu' au XVII ${ }^{\mathrm{e}}$ siècle les filles pouvaient recevoir leur nom soit de leurs marraines, soit de leurs parrains, qui leur transmettaient dans ce cas une forme féminisée de leur propre nom. Cela a un effet immédiat dans la typologie des répertoires féminins car on y constate alors une majorité de noms masculins féminisés (Guillemette, Perrine, Jacquette, etc.) et une faible proportion de noms de saintes femmes (Anne, Catherine, Marguerite, Marie, etc.). Cette pratique était la norme dans certaines régions françaises, notamment en Bretagne orientale, en Savoie et dans une partie de la Franche-Comté ${ }^{45}$. En

44. En Bretagne au XVI ${ }^{\mathrm{e}}$ siècle, les taux de transmission homonymique se situent sauf exceptions entre $55 \%$ et $90 \%$ à l'ouest et entre $80 \%$ et $95 \%$ à l'est. Dans le Rouergue, les taux sont de $93 \%$ pour les garçons et de $86 \%$ pour les filles à Cadour (1588-1648), de $95 \%$ pour les garçons et de $96 \%$ pour les filles à La Terrisse (1589-1645) mais seulement de $54 \%$ pour les garçons et $68 \%$ pour les filles à Luc (1580-1664). En Franche-Comté, les taux sont de $93 \%$ pour les garçons et de $91 \%$ pour les filles à Arbois (1552-1589) et de $92 \%$ pour les garçons et $87 \%$ pour les filles à Saint-Claude (1592-1599). En Savoie, les taux sont de $81 \%$ pour les garçons et $83 \%$ pour les filles à Belley (1574-1590) et de $83 \%$ pour les garçons et les filles à Brénod (1547-1569). Statistiques personnelles.

45. C'est le cas en Bretagne au XV ${ }^{\mathrm{e}}$ et XVI ${ }^{\mathrm{e}}$ siècle dans les évêchés de Saint-Malo, Dol et Rennes, ainsi que dans les paroisses franc-comtoises ou savoyardes situées dans la zone linguistique du franco-provençal (études 
basse Auvergne, la nomination des filles est presque toujours l'affaire des marraines ${ }^{46}$. Les attributions transversales sont peu fréquentes dans l'Ouest et, lorsqu'il y a transmission homonymique, c'est presque toujours le nom de la marraine qui est transmis à la filleule. Dans l'Est, les attributions transversales parrains/filleules sont généralement inexistantes ou rares dans le pays de Billom (Chauriat, Saint-Julien-de-Coppel) mais plus fréquentes dans le cœur du Livradois : $12 \%$ au Monestier, $34 \%$ à Tours-sur-Meymont. Cela peut signifier que les parrains y bénéficiaient d'un honneur plus marqué que dans les autres paroisses.

Le cas de Tours-sur-Meymont constitue une exception intéressante. Toutes les données recueillies contribuent en effet à en donner l'image d'une paroisse où la femme était moins valorisée qu'ailleurs : le taux des transmissions homonymiques est bien plus faible pour les filles $(86 \%)$ que pour les garçons $(95 \%)$, le nom Marie y est peu attribué $(5,6 \%$ des occurrences contre $10,8 \%$ au Monestier) et le modèle ternaire féminin typique du Livradois n'y était pas en usage. A contrario, la délégation fréquente de la nomination des filles aux parrains valorisait ces derniers. Le surcroît d'honneur conféré aux parrains se justifiait peut-être par le fait que les parents attendaient ici davantage d'assistance mutuelle de la part de leurs compères ou bien une participation financière plus importante dans les frais du baptême (ce qui pourrait expliquer l'absence de recours à une seconde marraine).

\section{Le parrainage intrafamilial}

Les analyses précédentes ont montré le poids des liens du compérage dans la société auvergnate du $\mathrm{XVI}^{\mathrm{e}}$ siècle et ont révélé des communautés fortement structurées, chargées de répondre aux besoins d'entraide, que ce soit dans les tâches quotidiennes ou au moment de crises plus intenses. Mais cette recherche de soutiens à la cellule familiale, à qui était-elle adressée : aux élites sociales de la communauté, aux voisins ou au milieu familial proche ? Pour déterminer l'ampleur du recours au parrainage intrafamilial, il existe une méthode de calcul approximative mais relativement simple à mettre en place : il s'agit de la méthode des homonymies patronymiques qui consiste à recenser tous les cas où le parrain ou la marraine portent le patronyme de l'un des parents. Ce critère est bien entendu incomplet puisqu'il ne recense qu'une partie de la parenté, notamment les grands-pères des nouveau-nés et leurs oncles et tantes biologiques, laissant de côté les grands-mères et les oncles et tantes par alliance ${ }^{47}$. En théorie, comme le nombre des seconds est approximativement le même que celui des premiers, la part du parrainage intrafamilial global pourrait logiquement être estimée au double du taux des homonymies patronymiques. Toutefois, il arrive que dans certaines régions comme le Rouergue les taux d'homonymies patronymiques soient proches de $55 \%$, alors qu'en tout état de cause, il ne peut y avoir un parrainage intrafamilial à $110 \%$. Dans une paroisse, on peut vraisemblablement estimer le taux maximum du parrainage intrafamilial à $90 \%$ (il y a toujours quelques exceptions), ce qui nous donne un

personnelles). Sur la pratique du parrainage en Bretagne à cette époque, voir Pierre-Yves Quémener, « Parrainage et nomination en Bretagne aux XV $\mathrm{VV}^{\mathrm{e}}$ et XVI $\mathrm{C}^{\mathrm{e}}$ siècles », Annales de démographie historique, 2017/1, p. 145-179.

46. L'analyse porte essentiellement sur les relevés de baptême du Puy-de-Dôme. Je ne dispose pas de données suffisantes pour établir des statistiques pour les paroisses de l'Allier.

47. À propos de la méthode des homonymies patronymiques, voir Camille Berteau, Vincent Gourdon et Isabelle Robin, « Trois siècles de parrainages à Aubervilliers. De la Réforme catholique au temps des banlieues industrielles », dans Le Parrainage en Europe et en Amérique. Pratiques de longue durée (XVI ${ }^{e}-X X I^{e}$ siècles), dir. Guido Alfani, Vincent Gourdon et Isabelle Robin, Bruxelles, Peter Lang, 2015, p. 49. 
coefficient approximatif de 1,65 entre le taux des homonymies patronymiques et celui taux du parrainage intrafamilial total ${ }^{48}$.

À l'exception du cas rouergat, les études publiées jusqu'à présent pour la période 15501650 affichent toutes des taux d'homonymies patronymiques situés entre $5 \%$ et $25 \%$, et plus généralement entre $10 \%$ et $20 \%$, que ce soit en Bretagne ${ }^{49}$, en Franche-Comté ${ }^{50}$, en Savoie ${ }^{51}$, en Île-de-France ${ }^{52}$, à Lyon ${ }^{53}$ ou à Bordeaux ${ }^{54}$. Le tableau 4 indique les taux d'homonymies patronymiques calculés pour quelques paroisses auvergnates.

Tab. 4 - Homonymies patronymiques de 1530 à 1630.

\begin{tabular}{|l|l|l|l|l|l|}
\hline \multicolumn{1}{|c|}{ Paroisse } & \multicolumn{1}{|c|}{ Période } & $\begin{array}{c}\text { Nbre } \\
\text { bapt. }\end{array}$ & $\begin{array}{l}\text { Homonymies } \\
\text { avec le père }\end{array}$ & $\begin{array}{l}\text { Homonymies } \\
\text { avec la mère }\end{array}$ & $\begin{array}{c}\text { Homonymies } \\
\text { Total }\end{array}$ \\
\hline Pontgibaud & $1550-1617$ & 1387 & $12,7 \%$ & $14,3 \%$ & $27,0 \%$ \\
\hline Herment & $1569-1599$ & 69 & $17,3 \%$ & $17,1 \%$ & $34,4 \%$ \\
\hline Besse & $1537-1599$ & 2481 & $16,5 \%$ & $18,0 \%$ & $34,5 \%$ \\
\hline Ardes & $1563-1632$ & 517 & $20,3 \%$ & $16,8 \%$ & $37,1 \%$ \\
\hline $\begin{array}{l}\text { Égliseneuve- } \\
\text { d'Entraigues }\end{array}$ & $1569-1606$ & 250 & $27,9 \%$ & $24,3 \%$ & $52,2 \%$ \\
\hline Condat & $1602-1630$ & 1307 & $24,0 \%$ & $24,1 \%$ & $48,1 \%$ \\
\hline Chauriat & $1578-1625$ & 450 & $17,3 \%$ & $18,6 \%$ & $35,9 \%$ \\
\hline $\begin{array}{l}\text { Tours-sur- } \\
\text { Meymont }\end{array}$ & $1577-1615$ & 1694 & $21,4 \%$ & $19,0 \%$ & $40,4 \%$ \\
\hline Le Monestier & $1599-1630$ & 862 & $31,6 \%$ & $25,0 \%$ & $56,6 \%$ \\
\hline
\end{tabular}

48. $90 / 55=1,64$. Ces observations résultent d'analyses personnelles effectuées dans diverses régions françaises. J'ai relevé dans le Rouergue des taux d'homonymies patronymiques particulièrement élevés au tournant du XVI ${ }^{\mathrm{e}}$ et du XVII ${ }^{\mathrm{e}}$ siècle : 54,6\% à Cadour en 1588-1648; 54,5\% à Coubisou en 1598-1650;51,8\% à La Terrisse en 1589-1645; 51,9\% à Luc en 1580-1664. Plusieurs sondages ont révélé que le parrainage intrafamilial était quasiment exclusif dans ces paroisses : 14 parrains et marraines issus de la parenté pour 7 naissances chez les Mazars de Luc entre 1594 et 1613 et un taux d'homonymies patronymiques de $64 \%$ (9/14); 8 parrains et marraines issus de la parenté pour 5 naissances dans la même paroisse chez les Arnal entre 1591 et 1603 avec un taux d'homonymies patronymiques de $50 \%(5 / 10)$. Les deux parrains non issus de la parenté étaient deux prêtres. Pour ces deux familles, le coefficient moyen entre la part du parrainage intrafamilial global et la part des homonymies patronymiques est de 1,6 (22/14). J'ai réalisé par ailleurs une étude généalogique sur une famille bretonne - les Gloanec de Gourin - portant sur un échantillon de 194 baptêmes enregistrés de 1611 à 1790. Pour toute cette période, j'ai comptabilisé 59 homonymies patronymiques (soit un taux de 15,2\% pour 388 parrains et marraines). Le décompte exact des parrains et marraines issus du milieu familial proche (grands-parents, oncles et tantes, cousins et cousines) donne un total de 108 apparentés, soit 27,8\%. Le coefficient entre la part du parrainage intrafamilial global et la part des homonymies patronymiques est de 1,8 .

49. 14,4 \% à Beignon en 1550-1599; 22,2 \% à Bignan en 1530-1591; 14,5\% à Plumelec en 1572-1599 ; $11,1 \%$ à Plumelec (statistiques personnelles).

50. $14,3 \%$ à Arbois en 1552-1589 (statistiques personnelles).

51. $14,4 \%$ à Hotonnes en 1600-1640 (statistiques personnelles).

52. 19,7 \% à Aubervilliers en 1552-1632 : C. Berteau, V. Gourdon et I. Robin, « Trois siècles de parrainage à Aubervilliers », art. cit., p. 51 .

53. 17,4\% dans la paroisse de Saint-Nizier en 1655 : Étienne Couriol, « La place de la parenté dans les baptêmes d'une paroisse lyonnaise d'Ancien Régime », dans Le Parrainage en Europe et en Amérique, op. cit., p. 295 .

54. $10,45 \%$ dans la paroisse de Saint-André en 1580 et $21,12 \%$ dans cette même paroisse en $1642 ; 8,63 \%$ dans la paroisse de Sainte-Croix et 9,32 \% dans celle de Saint-Seurin en 1642 : Stéphane Minvielle, « Baptême et parrainage à Bordeaux sous l'Ancien Régime », dans Le Parrainage en Europe et en Amérique, op. cit., p. $268-270$. 
Fig. 4 - Le parrainage intrafamilial dans la seconde moitié du XVI $\mathrm{I}^{\mathrm{e}}$ siècle.

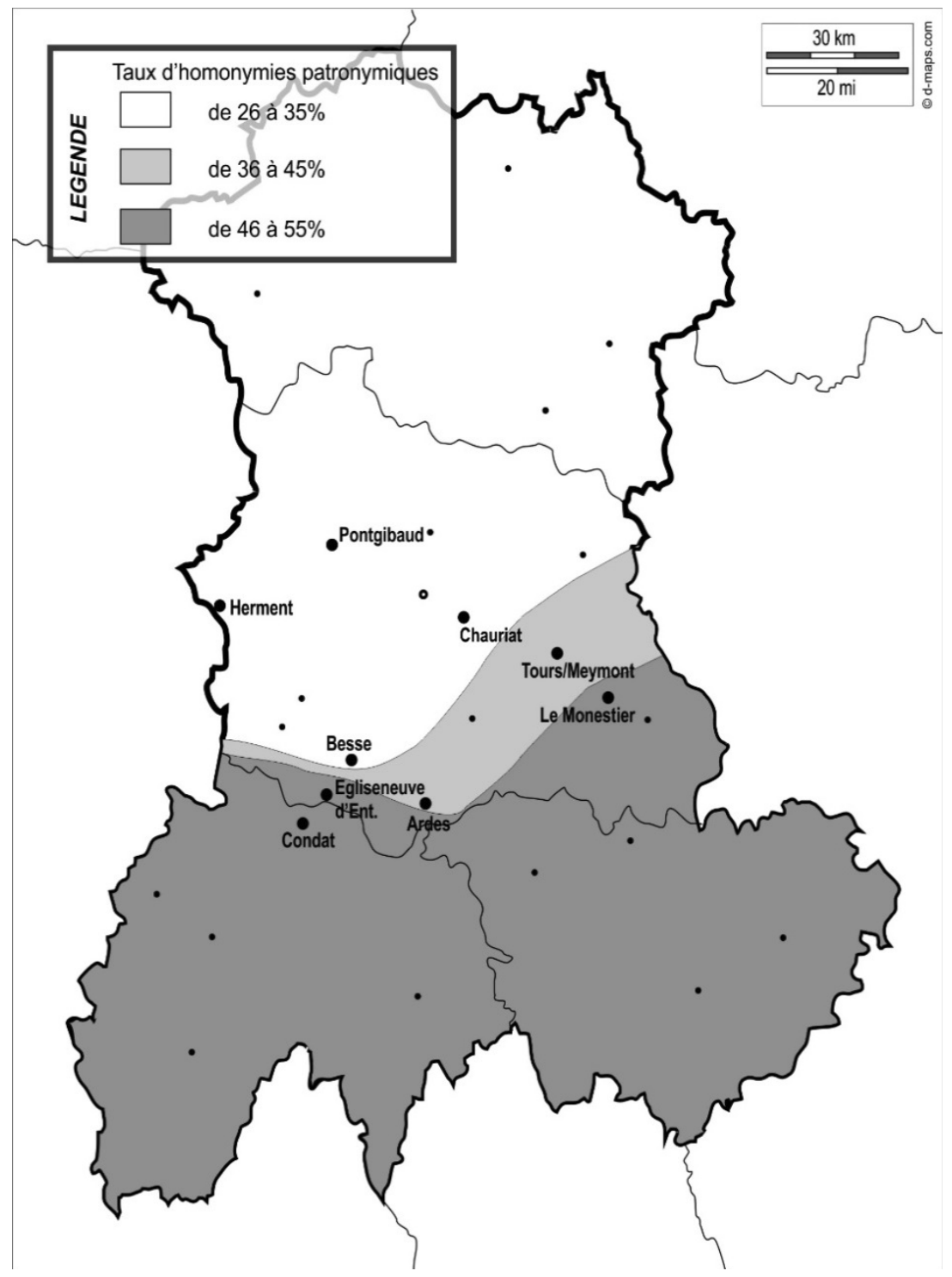

Le positionnement géographique des différentes localités (figure 4) met en évidence une progression des taux d'homonymies patronymiques au fur et à mesure que l'on descend vers le sud avec des maxima atteints dans l'Artense (Égliseneuve d'Entraigues, Condat) et dans le sud du Livradois (Le Monestier). Les données partielles collectées à Saint-Anthème $(22,1 \%)$ et à Saint-Clément-de-Valorgues $(22,9 \%)$ confirment les résultats ci-dessus car on ne connaît pour ces paroisses méridionales que les homonymies avec les pères (les mères ne sont pas nommées dans les actes). Il apparaît donc que dès la fin du XVI ${ }^{\mathrm{e}}$ siècle, 
le parrainage intrafamilial était quasiment exclusif dans le sud du Puy-de-Dôme et il est possible qu'il le fût depuis longtemps. Dans le Nord, le parrainage intrafamilial est prépondérant mais les familles sollicitent très régulièrement des parrains et marraines hors de la parenté. Nous nous trouvons manifestement encore dans une zone de transition où deux pratiques divergentes coexistent. Dans le Sud, les familles s'appuient essentiellement sur le soutien des proches parents dont on présume qu'ils vivaient généralement dans l'entourage immédiat. Cela peut expliquer le recours à un nombre minimum de parrains et marraines. Plus on remonte vers le Nord et plus les attentes en matière de solidarité se tournent vers le voisinage, peut-être par défaut, parce que les familles étaient plus dispersées, ou peut-être parce que les conceptions de la vie sociale elle-même - et la place de la famille dans la société - étaient perçues différemment.

Fig. 5 - Homonymies patronymiques à Besse, Pontgibaud et Tours-sur-Meymont.

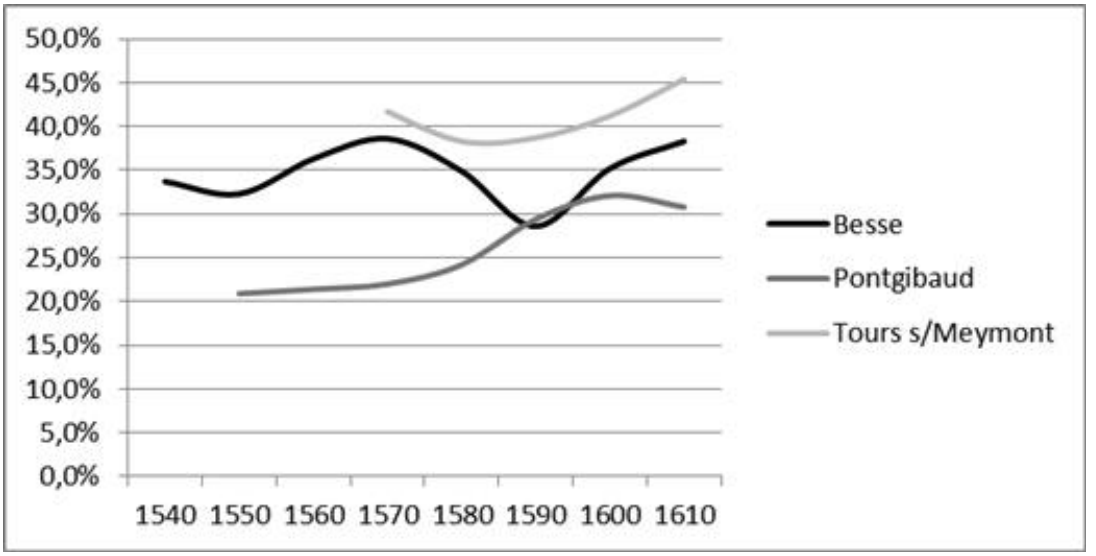

Du point de vue chronologique, les données des paroisses de Besse, Pontgibaud et Tourssur-Meymont (figure 5) révèlent des évolutions fluctuantes, avec toutefois une tendance générale à la hausse au tournant du XVI ${ }^{\mathrm{e}}$ et du XVII ${ }^{\mathrm{e}}$ siècle. À Besse, on observe cependant que le taux des homonymies patronymiques régresse fortement au moment des guerres de religion et l'histoire de la ville apporterait peut-être des clés d'interprétation de cette recrudescence du recours au parrainage extra-familial pendant cette période troublée.

En définitive, la basse Auvergne et le Bourbonnais offrent une étonnante diversité de pratiques du parrainage qui, sans coïncider tout à fait à la frontière linguistique, renvoient néanmoins à des formes d'organisation sociale différentes entre le nord, pays de langue d'oïl, et le sud, pays de langue d'oc. La forme la plus ancienne semble être celle que l'on retrouve dans le Sud. $\mathrm{Au} \mathrm{XVI} \mathrm{X}^{\mathrm{e}}$ siècle, la vie sociale y était fortement concentrée sur le milieu familial. On se contente d'un nombre minimum de parrains et marraines et le parrainage intrafamilial y est très développé, probablement parce que les familles vivaient depuis longtemps en proximité sur le même terroir. Les solidarités familiales y sont suffisamment solides pour se permettre de se passer d'un réseau de solidarité de voisinage étendu. Il est vraisemblable que cette forme d'organisation sociale était également prédominante en Auvergne septentrionale avant le $\mathrm{XV}^{\mathrm{e}}$ siècle, tout au moins dans le département actuel du Puy-de-Dôme, car on y décèle les traces d'une influence progressive des pratiques en usage dans les pays de langue d'oïl. L'hypothèse la plus probable est celle d'une acculturation 
qui se serait produite au cours du $X V^{\mathrm{e}}$ siècle, après les grandes crises démographiques, et consécutive à l'installation dans les massifs montagneux auvergnats de familles de défricheurs originaires du Nord, ne disposant pas à leur arrivée de réseaux familiaux étendus. Elles auraient apporté avec elles le modèle ternaire du parrainage caractéristique des pays de langue d'oïl et le recours prioritaire au parrainage extra-familial, indice d'un système social qui privilégiait les solidarités de voisinage, sans que cette nouvelle pratique abolisse toutefois le recours fréquent au parrainage de la parenté. Une particularité de la basse Auvergne est l'émergence de deux modèles tout à fait originaux dans la zone d'acculturation. Dans les Combrailles et la Chaîne des Puys s'est développé un modèle quaternaire à deux parrains et deux marraines. Dans les massifs du Livradois et du Forez, le modèle ternaire importé des pays de langue d'oïl a subi une inflexion significative en acceptant un seul parrain au baptême quel que soit le sexe de l'enfant. Il faut croire que les populations étaient fortement attachées à ces modèles de multi-parrainage car ils subsisteront en certains lieux jusque dans les années 1620, en dépit des prescriptions tridentines.

UNIVERSITÉ D’ANGERS 